(C) 2017 IEEE. Personal use of this material is permitted. Permission from IEEE must be obtained for all other uses, in any current or future media, including reprinting/republishing this material for advertising or promotional purposes, creating new collective works, for resale or redistribution to servers or lists, or reuse of any copyrighted component of this work in other works. 


\title{
Dimming Compatible Optical OFDM for High Speed Visible Light Communications
}

\author{
Thomas Q. Wang and Xiaojing Huang \\ Global Big Data Technologies Centre \\ University of Technology Sydney \\ Sydney, Australia \\ \{qian.wang, xiaojing.huang\}@uts.edu.au
}

\begin{abstract}
In this paper, we study a novel optical orthogonal frequency division multiplexing (OFDM) scheme, fractional reversed polarity optical OFDM (FRPO-OFDM), which enables dimmable visible light communications. The scheme combines an asymmetrically clipped optical OFDM (ACO-OFDM) sequence with an information-carrying brightness control sequence (BCS). We show that the new scheme can create a wide range of brightness levels whilst transmitting extra information using the BCS to enhance the spectral efficiency. The detector which can recover information from both sequences is derived based on maximum likelihood (ML) detection and estimation. It is shown that the information carried by the BCS using variable on-off keying (VOOK) can be detected with extremely low bit error rate (BER) and that the resulting overall BER of FRPO-OFDM is constant over a wide dimming range.
\end{abstract}

Keywords-ACO-OFDM; Digital Dimming Control; Variable On-Off Keying; Visible Light Communications

\section{INTRODUCTION}

With the increasing use of the energy efficient white light emitting diodes (LEDs), visible light communication (VLC) using intensity modulation direct detection (IM/DD) is emerging as a promising dual-use technique for indoor illumination and high speed data transmission. From the perspective of lighting, brightness control is essential to deliver a comfortable, energy efficient and aesthetic lighting experience. This, however, leads to a challenge from the perspective of data transmission, that is, the modulation techniques used by VLC must be dimming compatible [1].

Brightness control can be implemented in a number of ways. The most straightforward is known as the analog dimming which adjusts the brightness level through directly controlling the strength of the current input into the LEDs. This is possibly the simplest solution but may lead to a noticeable chromaticity shift [1]. Alternatively, digital dimming can be applied, in which rectangular pulses are transmitted and the brightness level is controlled through varying their duty cycle [2]. Typical digital dimming schemes include variable on-off keying (VOOK), variable pulse position modulation (VPPM) and multiple pulse position modulation (MPPM). Compared with analog dimming, these schemes provide more accurate control and induce less chromaticity shift. Therefore, they are preferred in industry standards.

This work was supported under an Australian Research Council's (ARC) Discovery funding scheme (DP 160101693).
Original optical orthogonal frequency division multiplexing (OFDM) techniques, such as asymmetrically clipped optical OFDM (ACO-OFDM) [3] and DC biased optical OFDM (DCO-OFDM) are not compatible with brightness control. This is because OFDM signals typically have high peak-to-average power ratio (PAPR) and the direct control of their DC components may lead to the peaks going beyond the dynamic range of the LEDs, resulting in (1) significant signal distortion and (2) insufficient utilization of the LED's dynamic range. To address this problem, dimmable OFDM methods are recently proposed using digital dimming $[4,5]$. In both of the methods, a periodic binary brightness control sequence (BCS) is employed to control the brightness with its duty cycle, but carries no information.

In this paper, we study an enhanced reverse polarity optical OFDM (RPO-OFDM), called fractional RPO-OFDM (FRPOOFDM), which transmits information using both the OFDM signal and the BCS to improve the spectral efficiency. The proposed OFDM scheme can be a combination of ACOOFDM with any digital dimming scheme [2]. Here we demonstrate its feasibility and focus on the case where VOOK is used. We use ACO-OFDM other than DCO-OFDM to ensure a reasonably wide brightness range [5]. We show that FRPO-OFDM can efficiently utilize the LED's dynamic range, delivering a wide dimming range. A high performance detector is also derived based on maximum likelihood (ML) estimation and detection. It is shown that, over a wide range of brightness, the extra information carried by the BCS can be decoded with extremely low bit error rate (BER). This leads to negligible impact of the these information bits on the demodulation of the ACO-OFDM signal and implies that the spectral efficiency can be further improved using higher order modulation such as $M$ arry pulse position modulation (M-PPM) in the BCS.

\section{Signal Design AND Dimming CONTROL}

\section{A. FRPO-OFDM}

In this section, we present the format of a FRPO-OFDM signal. FRPO-OFDM distinguishes from the original RPOOFDM [5] in two major aspects. First, the periodic BCS is replaced by a random information carrying sequence in the form of VOOK. Thus, the BCS used in FRPO-OFDM will have two possible waveforms each corresponding to a possible 
information bit. Second, each BCS is configured to have a size identical to that of an OFDM symbol. Therefore, unlike RPOOFDM [5], the transition between the on and off states in a BCS will be located within each OFDM symbol and the dimming level depends on the fractions the two states take up.

A FRPO-OFDM symbol is constructed using three modules: (1) an ACO-OFDM modulator [3], (2) a VOOK modulator [2] and (3) a combining module. The ACO-OFDM modulator converts complex bipolar symbols $\mathbf{X}=\left[0, X_{1}, 0, X_{3}, \ldots, X_{N-1}\right]^{T}$ to real non-negative sequences, $\mathbf{s}=\left[s_{0}, s_{1}, \ldots, s_{N-1}\right]^{T}$, through an intermediate sequence, $\mathbf{x}=\left[x_{0}, x_{1}, \ldots, x_{N-1}\right]^{T}$, where $\mathbf{x}$ is the inverse fast Fourier transform (IFFT) of $\mathbf{X}$ with electrical power $E\left(x_{n}^{2}\right)=\sigma_{\mathrm{x}}^{2}$, $n=0, \ldots, N-1$ and $\mathbf{s}$ is obtained by clipping $\mathbf{x}$ at zero. As both $\mathbf{x}$ and $\mathbf{s}$ are real, Hermitian symmetry is imposed at $\mathbf{X}$.

The BCS, $\mathbf{b}=\left[b_{0}, b_{1}, \ldots, b_{N-1}\right]^{T}$, is generated by the VOOK modulator, where $b_{n}=0$ and 1 correspond to the off and on states, respectively. In general, a BCS consists of two portions: an active portion with a length of $m$ bits which carries information and an inactive portion consisting of $N-m$ bits, used as filling bits for brightness control [2]. Thus, the duty cycle of the sequence, $D$, is given by $m / N$. Depending on the information to be transmitted, all the bits in the active portion take identical value equaling either one or zero.

The combining module performs an element-wise multiplication and addition on the two sequences, yielding the FRPO-OFDM signal given by

$$
y_{n}=p_{n}\left(-s_{n}^{(c)}+A\right)+I_{c}, \quad \text { for } 0 \leq n \leq N-1,
$$

where $, I_{\mathrm{c}}, I_{\mathrm{c}}>A$ and $2 A, A>0$, denote the center value and the width of the signal's dynamic range, respectively. $s_{n}^{(c)}$ is obtained by clipping $s_{n}$ at $2 A$, i.e.

$$
s_{n}^{(c)}= \begin{cases}s_{n}, & s_{n}<2 A \\ 2 A, & s_{n} \geq 2 A\end{cases}
$$

and sequence $\mathbf{p}=\left[p_{0}, p_{1}, \ldots, p_{N-1}\right]^{T}$ is related to the BCS by $p_{n}=2 b_{n}-1$.

As shown in (1), the term, $-s_{n}^{(c)}+A, 0 \leq n \leq N-1$, is a non-positive going sequence biased at $A$, representing the on state. Thus, the maximum and minimum values of this sequence will be $A$ and $-A$, respectively, i.e., this sequence is constrained between $\pm A$. When multiplied with $p_{n}$ which equals either 1 or -1 , the sequence will either remain at the on state or be flipped to the off state, resulting in the term, $p_{n}\left(-S_{n}^{(c)}+A\right)$, a sequence centered at 0 with a dynamic range of $2 A$. A DC bias, $I_{\mathrm{c}}$, is then superimposed, leading to the FRPO-OFDM signal ranging from $I_{\mathrm{L}}=I_{\mathrm{c}}-A$ to $I_{\mathrm{H}}=I_{\mathrm{c}}+A$. The resulting signal is emitted by the LEDs after adding a cyclic prefix and digital-to-analogue conversion.

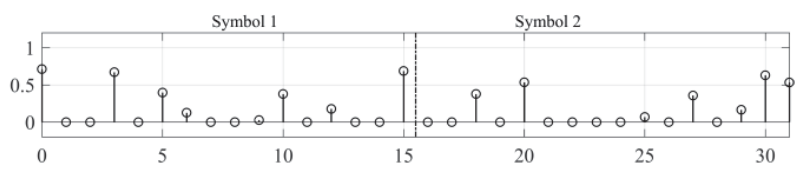

(a)

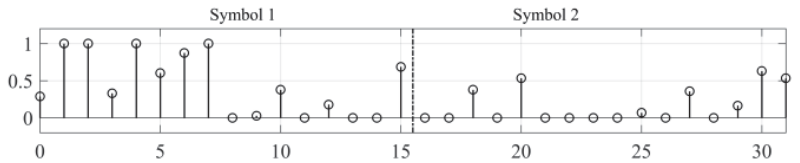

(b)

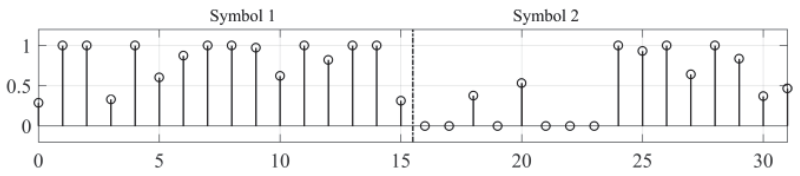

(c)

Fig. 1. A demonstration of the FRPO-OFDM signal.

Fig.1 demonstrates two successive FRPO-OFDM symbols, where Fig. 1(a) plots the original ACO-OFDM signal, and Fig. 1(b) and (c) the FRPO-OFDM signal using different filling bits. For Symbol 1, an information bit " 1 " is transmitted by the BCS and for Symbol 2, " 0 ". The duty cycle is set to be 0.5 and the active portion is located in the first half of the BCS. The filling bits, 0 and 1, are used in Fig. 1(b) and (c), respectively. Comparing Fig. 1(a) with (b) and (c), we can see that because of the use of a BCS, the average optical power of the FRPOOFDM is different from that of the original ACO-OFDM. This confirms that FRPO-OFDM can indeed adjust the average strength of the current. Also, the ACO-OFDM signal is seen completely contained in the dynamic range regardless of the brightness level. This addresses the issue raised in analog dimming. From Fig. 1(b) and (c), it can be seen that using "1" as filling bits can generate higher optical power than using " 0 ". This is consistent with the original VOOK.

\section{B. Measured and Perceived Brightness}

Now we derive the expression of the measured and perceived brightness. We assume the dynamic range of the FRPO-OFDM signal is included in that of the LED. Then the electrical-to-optical conversion is linear. In line with [5], the measured brightness level (MBL) is given by

$$
\mathrm{BL}_{\mathrm{m}}=\frac{I_{\mathrm{avg}}-I_{\mathrm{L}}}{I_{\mathrm{H}}-I_{\mathrm{L}}},
$$

where $I_{\text {avg }}$ denotes the average current input to the LED. Assuming the BCS transmits 1 and 0 with equal probability, as the average current equals $I_{\mathrm{H}}-\bar{S}_{n}^{(c)}$ and $I_{\mathrm{L}}+\bar{S}_{n}^{(c)}$ in the on and off states, respectively, $I_{\text {avg }}$ can be expressed as

$$
\begin{aligned}
I_{\text {avg }}= & \frac{1}{2}\left\{\frac{q_{0,1}}{N}\left(I_{\mathrm{H}}-\bar{s}_{n}^{(c)}\right)+\frac{q_{0,0}}{N}\left(I_{\mathrm{L}}+\bar{s}_{n}^{(c)}\right)\right\} \\
& +\frac{1}{2}\left\{\frac{q_{1,1}}{N}\left(I_{\mathrm{H}}-\bar{s}_{n}^{(c)}\right)+\frac{q_{1,0}}{N}\left(I_{\mathrm{L}}+\bar{s}_{n}^{(c)}\right)\right\},
\end{aligned}
$$


where $q_{k_{1}, k_{2}}, k_{1}, k_{2}=0,1$, denotes the number of $k_{2} \mathrm{~s}$ in the BCS when $k_{1}$ is transmitted and $\bar{s}_{n}^{(c)}$ is the expectation of $s_{n}^{(c)}$. As $s_{n}^{(c)}$ is a truncated Gaussian random variable which equals zero and $2 A$ with probability of 0.5 and $Q\left(2 A / \sqrt{\sigma_{\mathrm{x}}}\right)$, respectively, and follows Gaussian distribution in between, where $Q\left(\right.$.) denotes the Gaussian Q function, $\bar{s}_{n}^{(c)}$ is given by

$$
\begin{aligned}
\bar{S}_{n}^{(c)} & =0 \times 0.5+\int_{0}^{2 A} \frac{s}{\sqrt{2 \pi} \sigma_{\mathrm{x}}} \exp \left(-\frac{s^{2}}{2 \sigma_{x}^{2}}\right) \mathrm{d} s+2 A Q\left(\frac{2 A}{\sqrt{\sigma_{\mathrm{x}}}}\right) \\
& =2 A Q\left(\frac{2 A}{\sqrt{\sigma_{\mathrm{x}}}}\right)+\frac{\sigma_{\mathrm{x}}}{\sqrt{2 \pi}}\left(1-\exp \left(-\frac{2 A^{2}}{\sigma_{\mathrm{x}}^{2}}\right)\right) .
\end{aligned}
$$

The value of $q_{k_{1}, k_{2}}$ depends on the duty cycle and filling bits of the BCS. For the case where all the filling bits are zeros, the BCS transmitting bit 0 are $N 0 \mathrm{~s}$, leading to $q_{0,1}=0, q_{0,0}=N$ $q_{1,1}=D N$, and $q_{1,0}=(1-D) N$. This simplifies (4) as

$$
I_{\text {avg }}=I_{L}+\bar{s}_{n}^{(c)}+\frac{1}{2} D\left(I_{H}-I_{L}-2 \bar{s}_{n}^{(c)}\right), \quad \text { filling bit }=0 .
$$

Otherwise, the BCS transmitting bit 1 consists of $N$ 1s , resulting in $q_{0,1}=(1-D) N, q_{0,0}=D N, q_{1,1}=N$, and $q_{1,0}=0$. The average current can thus be rewritten as

$$
I_{\text {avg }}=I_{\mathrm{H}}-\bar{S}_{n}^{(c)}-\frac{1}{2} D\left(I_{H}-I_{L}-2 \bar{S}_{n}^{(c)}\right), \quad \text { filling bit }=1 .
$$

The perceived brightness level (PBL), $\mathrm{BL}_{\mathrm{p}}$, is related to the MBL by $\mathrm{BL}_{\mathrm{p}}=\sqrt{\mathrm{BL}_{\mathrm{m}}}[5]$.

\section{SignAL DETECTION}

In this section, we derive the detector that recovers the information from both the OFDM signal and the BSC. Because of the DC offset existing between the transmitter and receiver [6], the received signal can be expressed, in a compact form, as

$$
\mathbf{z}=-\mathbf{p} \otimes \mathbf{s}^{(c)}+A \mathbf{p}+I_{c}^{\prime} \mathbf{1}+\mathbf{w},
$$

where " $\otimes$ " denotes the element-wise multiplication, and $I_{c}^{\prime}$, $\mathbf{s}^{(c)}=\left[s_{0}^{(c)}, s_{1}^{(c)}, \ldots, s_{N-1}^{(c)}\right]^{T}, \quad \mathbf{w}=\left[w_{0}, w_{1}, \ldots, w_{N-1}\right]^{T}$ and $\mathbf{1}$ represent the DC-bias at the receiver, the vectors of the clipped OFDM samples, the noise and ones respectively. In this paper, only shot noise is considered, which is induced by the ambient light. Thus $\mathbf{w}$ is Gaussian distributed having independent elements with power of $\sigma_{\mathrm{w}}^{2}$.

As the relationship $\mathbf{p} \otimes \mathbf{p}=\mathbf{1}$ holds, if the information carried by the BCS is correctly decoded, multiplying the decoded $\mathbf{p}$ with the received signal yields

$$
-\mathbf{p z}=\mathbf{s}^{(c)}-A \mathbf{1}-I_{\mathbf{c}}^{\prime} \mathbf{p}-\mathbf{p w} .
$$

From (9), we can see that the information symbols are carried on the odd subcarriers of $\mathbf{s}^{(c)}$, whereas the term, $A \mathbf{1}$, has only DC component and the spectrum of $-I_{\mathbf{c}}^{\prime} \mathbf{p}$ depends on the property of $\mathbf{p}$. In this paper, in order to avoid the interference, the BCS as well as vector $\mathbf{p}$ is assumed to be repetitive, i.e. $b_{n}=b_{N / 2+n}, 0 \leq n \leq N / 2-1$. Then the fast Fourier transform (FFT) of $\mathbf{p}$ will be nonzero only on the even subcarriers.

\section{A. Estimation of the DC Bias}

The decoding of the VOOK signal can be modeled as a binary hypothesis testing with both random $\left(\mathbf{s}^{(c)}\right)$ and deterministic $\left(I_{\mathrm{c}}^{\prime}\right)$ nuisance parameters. We denote the two hypotheses by $H_{0}$ and $H_{1}$, where 0 and 1 are transmitted by the BCS using $\mathbf{b}^{H_{0}}$ and $\mathbf{b}^{H_{1}}$, respectively. The vector $\mathbf{p s}$ corresponding to the two possible sequences are then given by $\mathbf{p}^{H_{0}}=\left[p_{0}^{H_{0}}, \ldots, p_{N-1}^{H_{0}}\right]^{T}$ and $\mathbf{p}^{H_{1}}=\left[p_{0}^{H_{1}}, \ldots, p_{N-1}^{H_{1}}\right]^{T}$, respectively. We define two auxiliary vectors given by $\mathbf{u}^{H_{i}}=\mathbf{z}-A \mathbf{p}^{H_{i}}$. Then, conditioned on $I_{\mathrm{c}}^{\prime}, \mathbf{s}^{(c)}$ and $H_{i}, i=0,1$, the joint probability density function (PDF) of $\mathbf{u}^{H_{i}}$ can be expressed as

$$
\begin{aligned}
& p_{\mathbf{u}^{H_{i}}}\left(u_{0}^{H_{i}}, \ldots, u_{N-1}^{H_{i}} \mid I_{\mathrm{c}}^{\prime}, \mathbf{s}^{(c)}, H_{i}\right) \\
= & \left(\frac{1}{\sqrt{2 \pi} \sigma_{\mathrm{w}}}\right)^{N} \exp \left[-\frac{1}{2 \sigma_{\mathrm{w}}^{2}} \sum_{n=0}^{N-1}\left(u_{n}^{H_{i}}+p_{n}^{H_{i}} s_{n}^{(c)}-I_{\mathrm{c}}^{\prime}\right)^{2}\right] .
\end{aligned}
$$

Conditioned on hypothesis $H_{i}$, the ML estimate of $\mathbf{s}^{(c)}$ and $I_{\mathrm{c}}^{\prime}, \hat{\mathbf{s}}_{H_{i}}^{(c)}$ and $\hat{I}_{\mathrm{c}}^{H_{i}}$, can be derived by taking the logarithm on (10), differentiating it with respect to $\mathbf{s}^{(c)}$ and $I_{\mathrm{c}}^{\prime}$, and forcing the result to zero to give

$$
\left[\begin{array}{ccccc}
N & -p_{0}^{H_{i}} & -p_{1}^{H_{i}} & . . & -p_{N-1}^{H_{i}} \\
-1 & p_{0}^{H_{i}} & 0 & . . & 0 \\
-1 & 0 & p_{1}^{H_{i}} & . . & 0 \\
: & : & : & & : \\
-1 & 0 & 0 & . . & p_{N-1}^{H_{i}}
\end{array}\right]\left[\begin{array}{c}
\hat{I}_{\mathrm{c}}^{H_{i}} \\
\hat{s}_{0, H_{i}}^{(c)} \\
\hat{s}_{1, H_{i}}^{(c)} \\
: \\
\hat{s}_{N-1, H_{i}}^{(c)}
\end{array}\right]=\left[\begin{array}{c}
\sum_{n=0}^{N-1} u_{n} \\
-u_{0}^{H_{i}} \\
-u_{1}^{H_{i}} \\
\vdots \\
-u_{N-1}^{H_{i}}
\end{array}\right] .
$$

As $\mathbf{s}^{(c)}$ can be obtained by clipping an intermediate sequence, $\mathbf{x}^{(c)}$, which is anti-periodic, i.e. $x_{n}^{(c)}=-x_{N / 2+n}^{(c)}$, where $\mathbf{x}^{(c)}$ is obtained by clipping $\mathbf{x}$ at $\pm 2 A, s_{n}^{(c)}$ and $s_{N / 2+n}^{(c)}$ can be expressed in term of $x_{n}^{(c)}$ as

$$
s_{n}^{(c)}=\left(x_{n}^{(c)}+\left|x_{n}^{(c)}\right|\right) / 2, s_{N / 2+n}^{(c)}=\left(-x_{n}^{(c)}+\left|x_{n}^{(c)}\right|\right) / 2 .
$$

Thus, to comply with (12), the estimate, $\hat{\mathbf{s}}_{H_{i}}^{(c)}$, can be formed as

$$
\hat{s}_{n, H_{i}}^{(c)}=\left(\hat{x}_{n, H_{i}}^{(c)}+\left|\hat{x}_{n, H_{i}}^{(c)}\right|\right) / 2, \hat{s}_{N / 2+n}^{(c)}=\left(-\hat{x}_{n, H_{i}}^{(c)}+\left|\hat{x}_{n, H_{i}}^{(c)}\right|\right) / 2,
$$


where $\hat{x}_{n, H_{i}}^{(c)}$ is the estimate of $x_{n}^{(c)}$ conditioned on $H_{i}$.

Substituting (13) into (11), the estimate of the DC bias, $\hat{I}_{\mathrm{c}}^{H_{i}}$, can be expressed as

$$
\hat{I}_{\mathrm{c}}^{H_{i}}=\frac{1}{N} \sum_{n=0}^{N-1}\left(p_{n}^{H_{i}} \hat{S}_{n, H_{i}}^{(c)}+u_{n}^{H_{i}}\right)
$$

where for $0 \leq n \leq N / 2-1$,

$$
\begin{aligned}
& \hat{s}_{n, H_{i}}^{(c)}=\left(\hat{x}_{n, H_{i}}^{(c)}+\left|\hat{x}_{n, H_{i}}^{(c)}\right|\right) / 2, \hat{x}_{n, H_{i}}^{(c)}=\left(u_{N / 2+n}^{H_{i}}-u_{n}^{H_{i}}\right) / p_{n}^{H_{i}}, \\
& \text { and } \hat{x}_{N / 2+n, H_{i}}^{(c)}=-\hat{x}_{n, H_{i}}^{(c)},
\end{aligned}
$$

\section{B. Detection of the VOOK Signal}

In this section, we develop a detector based on composite ML detection which calculates the likelihood ratio

$$
\Lambda=\frac{E_{x_{0}^{(c)}, \ldots, x_{N / 2-1}^{(c)}}\left(p_{\mathbf{u}^{H_{1}}}\left(u_{0}^{H_{1}}, \ldots, u_{N-1}^{H_{1}} \mid \hat{I}_{\mathrm{c}}^{H_{1}}, \mathbf{x}^{(c)}, H_{1}\right)\right)}{E_{x_{0}^{(c)}, \ldots, x_{N / 2-1}^{(c)}}\left(p_{\mathbf{u}^{H_{0}}}\left(u_{0}^{H_{0}}, \ldots, u_{N-1}^{H_{0}} \mid \hat{I}_{\mathrm{c}}^{H_{0}}, \mathbf{x}^{(c)}, H_{0}\right)\right)}
$$

and compares it with 1 , where the dc bias, $I_{\mathrm{c}}^{\prime}$, is substituted by its estimate in both hypotheses. Because of (12), the expectation is only taken over $x_{0}^{(c)}, \ldots, x_{N / 2-1}^{(c)}$. Using the periodicity of $\mathbf{p}$, the conditional PDF can be expressed as

$$
\begin{aligned}
& p_{\mathbf{u}^{H_{i}}}\left(u_{0}^{H_{i}}, \ldots, u_{N-1}^{H_{i}} \mid \hat{I}_{\mathrm{c}}^{H_{i}}, \mathbf{x}^{(c)}, H_{i}\right) \\
= & \prod_{n=0}^{N / 2-1} p_{u_{n}^{H_{i}}, u_{N / 2+n}^{H_{i}}}\left(u_{n}^{H_{i}}, u_{N / 2+n}^{H_{i}} \mid \hat{I}_{\mathrm{c}}^{H_{i}}, x_{n}^{(c)}, H_{i}\right) \\
= & \left(\frac{1}{\sqrt{2 \pi} \sigma_{\mathrm{w}}}\right)^{N} \prod_{n=0}^{N / 2-1} \exp \left\{-\frac{1}{2 \sigma_{\mathrm{w}}^{2}}\left[\left(u_{n}^{H_{i}}+0.5 p_{n}^{H_{i}}\left(x_{n}^{(c)}+\left|x_{n}^{(c)}\right|\right)-\hat{I}_{\mathrm{c}}^{H_{i}}\right)^{2}\right.\right. \\
& \left.\left.+\left(u_{N / 2+n}^{H_{i}}-0.5 p_{N / 2+n}^{H_{i}}\left(x_{n}^{(c)}-\left|x_{n}^{(c)}\right|\right)-\hat{I}_{\mathrm{c}}^{H_{i}}\right)^{2}\right]\right\} \\
= & \left(\frac{1}{\sqrt{2 \pi} \sigma_{\mathrm{w}}}\right)^{N} \prod_{n=0}^{N / 2-1} \exp \left\{-\frac{1}{2 \sigma_{\mathrm{w}}^{2}}\left[\left(x_{n}^{(c)}\right)^{2}+\left(u_{n}^{H_{i}}-u_{N / 2+n}^{H_{i}}\right) p_{n}^{H_{i}} x_{n}^{(c)}\right.\right. \\
& +\left(u_{n}^{H_{i}}+u_{N / 2+n}^{H_{i}}-2 \hat{I}_{\mathrm{c}}^{H_{i}}\right) p_{n}^{H_{i}}\left|x_{n}^{(c)}\right|+\left(u_{n}^{H_{i}}-\hat{I}_{\mathrm{c}}^{H_{i}}\right)^{2} \\
& \left.\left.+\left(u_{N / 2+n}^{H_{i}}-\hat{I}_{\mathrm{c}}^{H_{i}}\right)^{2}\right]\right\} .
\end{aligned}
$$

The joint PDF of $x_{0}^{(c)}, \ldots, x_{N / 2-1}^{(c)}$ depends on the distribution of $x_{0}, \ldots, x_{N / 2-1}$ which are Gaussian variables when the size of the IFFT, $N$, is large enough. Using the Hermitian symmetry, the output of the IFFT is given by [7]

$$
x_{n}=\frac{2}{\sqrt{N}} \sum_{k=1,3, \ldots}^{N / 2-1}\left(a_{k} \cos \frac{2 \pi k n}{N}-b_{k} \sin \frac{2 \pi k n}{N}\right),
$$

where $X_{k}=a_{k}+j b_{k}$ is a $M$-QAM symbol with average energy $E\left|X_{k}\right|^{2}=\varepsilon$. Assuming the symbols loaded on subcarriers are independent with identical energy, the correlation between samples can be expressed as

$$
\begin{aligned}
E\left(x_{m} x_{n}\right)= & E\left\{\frac{4}{N} \sum_{k_{1}=1,3, \ldots}^{N / 2-1} \sum_{k_{2}=1,3, \ldots}^{N / 2-1}\left(a_{k_{1}} \cos \frac{2 \pi k_{1} m}{N}-b_{k_{1}} \sin \frac{2 \pi k_{1} m}{N}\right)\right. \\
& \left.\times\left(a_{k_{2}} \cos \frac{2 \pi k_{2} n}{N}-b_{k_{2}} \sin \frac{2 \pi k_{2} n}{N}\right)\right\} \\
= & \frac{2 \varepsilon}{N} \sum_{k=1,3, \ldots}^{N / 2-1} \cos \frac{2 \pi}{N} k(n-m) \\
= & \left\{\begin{array}{cc}
\varepsilon / 2, & n=m \\
-\varepsilon / 2, & |n-m|=N / 2 \\
0, & \text { otherwise }
\end{array}\right.
\end{aligned}
$$

where the second equation holds as the $M$-QAM symbols have zero mean and their real and imaginary parts are independent. Eq. (19) confirms that the outputs of the IFFT have antiperiodic symmetry, and implies that they have identical power, $\sigma_{\mathrm{x}}^{2}=\varepsilon / 2$, and are independent of each other apart from those separated by $N / 2$ samples. As $x_{n}^{(c)}$ is a clipped version of $x_{n}, x_{0}^{(c)}, \ldots, x_{N / 2-1}^{(c)}$ are independent, equaling $\pm 2 A$ with the same probability of $Q\left(2 A / \sigma_{\mathrm{x}}\right)$ and following Gaussian distribution in between. Thus the PDF of $x_{n}^{(c)}$ is given by

$$
\begin{aligned}
p_{x_{n}^{(c)}}\left(x_{n}^{(c)}\right)= & Q\left(2 A / \sigma_{\mathrm{x}}\right) \delta(-2 A)+Q\left(2 A / \sigma_{\mathrm{x}}\right) \delta(2 A) \\
& +\frac{1}{\sqrt{\pi \varepsilon}} \exp \left(-\frac{\left(x_{n}^{(c)}\right)^{2}}{\varepsilon}\right) \operatorname{rect}(2 A),
\end{aligned}
$$

where $\delta($.$) is the Kronecker delta function and \operatorname{rect}(2 A)$ the rectangular function equaling one between $-2 A$ and $2 A$ and zero elsewhere.

The expectation in (16) can thus be expressed as

$$
\begin{aligned}
& E_{x_{0}^{(c)}, \ldots, x_{N / 2-1}^{(c)}}\left(p_{\mathbf{u}^{H_{i}}}\left(u_{0}^{H_{i}}, \ldots, u_{N-1}^{H_{i}} \mid \hat{I}_{\mathrm{c}}^{H_{i}}, \mathbf{x}^{(c)}, H_{i}\right)\right) \\
&= \underbrace{N / 2-1}_{n=0} Q\left(2 A / \sigma_{\mathrm{x}}\right) p_{u_{n}^{H_{i}}, u_{N / 2+n}^{H_{i}}}\left(u_{n}^{H_{i}}, u_{N / 2+n}^{H_{i}} \mid \hat{I}_{\mathrm{c}}^{H_{i}}, x_{n}^{(c)}=2 A, H_{i}\right) \\
&+\prod_{n=0}^{\prod_{1}^{H_{i}}} Q\left(2 A / \sigma_{\mathrm{x}}\right) p_{u_{n}^{H_{i}}, u_{N / 2+n}^{H_{i}}}\left(u_{n}^{H_{i}}, u_{N / 2+n}^{H_{i}} \mid \hat{I}_{\mathrm{c}}^{H_{i}}, x_{n}^{(c)}=-2 A, H_{i}\right) \\
&+\int_{-2, J_{2}^{H_{i}}}^{2 A} \underbrace{\underbrace{\prod_{i}}_{J_{3}^{H_{i}}}}_{\prod_{n=0}^{N / 2-1} p_{u_{n}^{H_{i}}, u_{N / 2+n}^{H_{i}}}\left(u_{n}^{H_{i}}, u_{N / 2+n}^{H_{i}} \mid \hat{I}_{\mathrm{c}}^{H_{i}}, x_{n}^{(c)}, H_{i}\right) p_{x_{n}^{(c)}}\left(x_{n}^{(c)}\right) \mathrm{d} x_{n}^{(c)}}
\end{aligned}
$$

where $J_{1}^{H_{i}}$ and $J_{2}^{H_{i}}$ are given by 


$$
\begin{aligned}
& J_{1}^{H_{i}}=\left(\frac{1}{\sqrt{2 \pi} \sigma_{\mathrm{w}}}\right)^{N} \prod_{n=0}^{N / 2-1} \exp \left\{-\frac{1}{2 \sigma_{\mathrm{w}}^{2}}\left[\left(u_{n}^{H_{i}}-\hat{I}_{\mathrm{c}}^{H_{i}}+2 A p_{n}^{H_{i}}\right)^{2}\right.\right. \\
&\left.\left.+\left(u_{N / 2+n}^{H_{i}}-\hat{I}_{\mathrm{c}}^{H_{i}}\right)^{2}\right]\right\} Q\left(2 A / \sigma_{\mathrm{x}}\right)
\end{aligned}
$$

and

$$
\begin{aligned}
J_{2}^{H_{i}}=\left(\frac{1}{\sqrt{2 \pi} \sigma_{\mathrm{w}}}\right)^{N} \prod_{n=0}^{N / 2-1} \exp \left\{-\frac{1}{2 \sigma_{\mathrm{w}}^{2}}\left[\left(u_{n}^{H_{i}}-\hat{I}_{\mathrm{c}}^{H_{i}}\right)^{2}\right.\right. \\
\left.\left.+\left(u_{N / 2+n}^{H_{i}}-\hat{I}_{\mathrm{c}}^{H_{i}}+2 A p_{n}^{H_{i}}\right)^{2}\right]\right\} Q\left(2 A / \sigma_{\mathrm{x}}\right) .
\end{aligned}
$$

Using Eq. (3.3221) of [8], $J_{3}^{H_{i}}$ can be expressed in a closed form as

$$
\begin{aligned}
J_{3}^{H_{i}} & =\left(\frac{1}{\sqrt{2 \pi} \sigma_{\mathrm{w}}}\right)^{N}\left(\frac{\beta}{\varepsilon}\right)^{N / 4} \\
& \times \prod_{n=0}^{N / 2-1} \exp \left\{-\frac{1}{2 \sigma_{\mathrm{w}}^{2}}\left[\left(u_{n}^{H_{i}}-\hat{I}_{\mathrm{c}}^{H_{i}}\right)^{2}+\left(u_{N / 2+n}^{H_{i}}-\hat{I}_{\mathrm{c}}^{H_{i}}\right)^{2}\right]\right\} \\
& \times\left\{\exp \left(\beta\left(\gamma_{n}^{H_{i}}\right)^{2}\right)\left[\Phi\left(\gamma_{n}^{H_{i}} \sqrt{\beta}+\frac{A}{\sqrt{\beta}}\right)-\Phi\left(\gamma_{n}^{H_{i}} \sqrt{\beta}\right)\right]\right. \\
& \left.+\exp \left(\beta\left(\gamma_{N / 2+n}^{H_{i}}\right)^{2}\right)\left[\Phi\left(\gamma_{N / 2+n}^{H_{i}} \sqrt{\beta}+\frac{A}{\sqrt{\beta}}\right)-\Phi\left(\gamma_{N / 2+n}^{H_{i}} \sqrt{\beta}\right)\right]\right\},
\end{aligned}
$$

where $\Phi($.$) denotes the Gauss error function and$

$$
\frac{1}{4 \beta}=\frac{1}{2 \sigma_{\mathrm{w}}^{2}}+\frac{1}{\varepsilon}, \quad \gamma_{n}^{H_{i}}=\frac{1}{\sigma_{\mathrm{w}}^{2}}\left(u_{n}^{H_{i}}-\hat{I}_{\mathrm{c}}^{H_{i}}\right) p_{n}^{H_{i}} .
$$

\section{Detection of the ACO-OFDM Signal}

Assuming the VOOK signal is decoded correctly, multiplying the corresponding $\mathbf{p}$ with $\mathbf{z}$ yields (9). As the interference term, $-A \mathbf{1}-I_{\mathbf{c}}^{\prime} \mathbf{p}$, has nonzero components only on the even subcarriers whereas the information symbols are loaded on the odd subcarriers of $\mathbf{s}^{(c)}$, the symbols to be decoded are free of interference. Therefore, classic decoding method can be used for the ACO-OFDM signal.

\section{SimULATION RESUlTS}

In this section, we present the simulation results for both dimming and BER performance that can be achieved by FRPO-OFDM. The dynamic range of the signal is configured to be $I_{\mathrm{L}}=0$ and $I_{\mathrm{H}}=1 \mathrm{~A}$ which are assumed to be identical to that of the LED. The ACO-OFDM modulator modulates 16QAM symbols onto $N=256$ subcarriers. The bandwidth of the FRPO-OFDM signal is set to be less than the modulation bandwidth of the LED, so the electrical-to-optical conversion factor in the LED is assumed to be a constant on all the subcarriers. The BER is simulated using 100000 FRPO-OFDM symbols that contain $25.6 \mathrm{M}$ bits in the ACO-OFDM symbols and $100 \mathrm{k}$ bits in the BCS. In line with [5], the noise power, $\sigma_{\mathrm{w}}^{2}$, is assumed at $-3 \mathrm{dBm}$.

\section{A. Measured and Perceived Brightness}

As shown in (3)-(7), the achieved brightness level is a function of the duty cycle, the average power, $\sigma_{x}^{2}$, and the fitting bits. Fig. 2 illustrates the interactions between these parameters and the brightness level where the probability shown in the legends denote that of the ACO-OFDM sequence, $\mathbf{s}$, clipped by the boundaries of the dynamic range, $I_{\mathrm{L}}$ and $I_{\mathrm{H}}$. The probability, 0.01 and 0.1 , correspond to $\sigma_{\mathrm{x}}^{2}$ equaling $15.3 \mathrm{dBm}$ and $25.7 \mathrm{dBm}$, respectively. As shown in Fig. 2(a), the fitting bits determine a coarse region the measured brightness level falls into. The all-one fitting bits lift the bright level above $50 \%$ whereas the brightness level falls into the lower half when the all-zero fitting bits are used. For either fitting bits, the $50 \%$ brightness level is achieved when the duty cycle, $D$, equals 1 . This is because for a unit duty cycle, the possible BSC sequence is either an all-one or an all-zero sequence, each with a probability of 0.5 . Fig. 2(a) also shows that the duty cycle of the BSC can provide a fine linear adjustment for the MBL in each region. This is desirable in the lighting industry. Fig. 2(b) shows the PBL. As shown in the figure, the light perceived is always brighter than that measured. This is due to the nonlinear relationship between the MBL and PBL caused by the adjustment of pupil size.

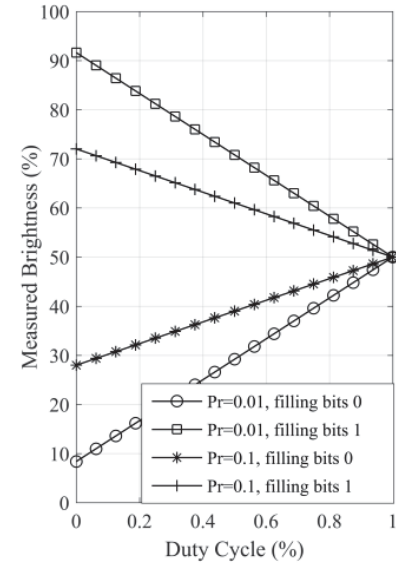

(a)

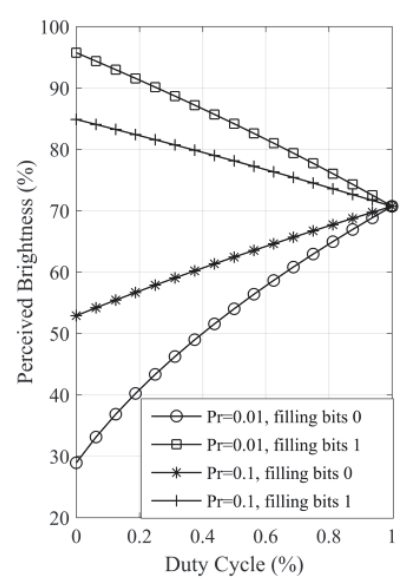

(b)
Fig. 2. Brightness level versus duty cycle.

It can be seen that the impact of power, $\sigma_{x}^{2}$, on the measured brightness level is reflected through the overall range of the provided brightness and the sensitivity of the brightness level to the duty cycle. As shown in the figure, an ACO-OFDM signal with $\sigma_{\mathrm{x}}^{2}=15.3 \mathrm{dBm}$ leads to MBLs ranging from $10 \%$ to $90 \%$, whereas $25.7 \mathrm{dBm}$ only from $30 \%$ to $70 \%$. A high power (i.e., high probability of clipping) produces reduced dimming range. This is because, as shown in (5), high power will generate high $\bar{s}_{n}^{(c)}$ which tends to lift up the off state and 
close down the on state of the BSC as given by (4). Also, as the MBLs always equal $50 \%$ at the duty cycle of 1 , the MBL with large $\sigma_{\mathrm{x}}^{2}$ will varies slowly as a function of the duty cycle, causing a low sensitivity.

\section{B. BER Performance}

Apart from the impact on the brightness level, $\sigma_{\mathrm{x}}^{2}$ also has a complex impact on the BERs of the VOOK and ACO-OFDM signals. First, as the VOOK signal is detected using composite ML detection which takes the ACO-OFDM signal as random nuisance parameters with power related to $\sigma_{\mathrm{x}}^{2}$, the increase of $\sigma_{\mathrm{x}}^{2}$ will degrade the detection performance, resulting in increasing BER for the VOOK signal and the error propagation to the decoding of the ACO-OFDM signal. Second, because the energy of the information symbols carried by the ACOOFDM signal is proportional to $\sigma_{x}^{2}$, when the nonlinear distortion caused by signal clipping is not dominating, the increase of $\sigma_{\mathrm{x}}^{2}$ will improve the BER of these symbols. Third, as $\sigma_{x}^{2}$ grows continuously, the high PAPR of the OFDM signals causing frequent signal clippings will become dominating. This along with the high BER of the VOOK signal will dramatically degrade the BER for both VOOK and ACOOFDM signals.

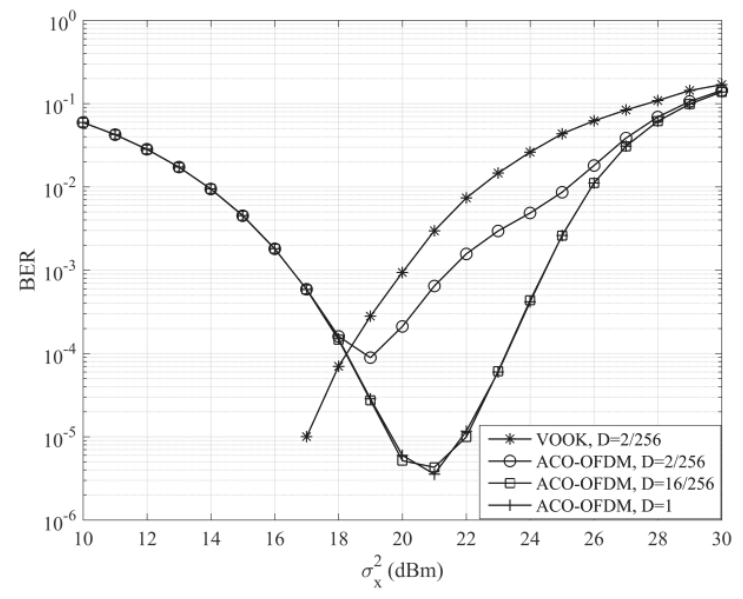

Fig. 3. BER versus $\sigma_{\mathrm{x}}^{2}$.

Fig. 3 shows the BERs for the VOOK and ACO-OFDM signals as functions of $\sigma_{\mathrm{x}}^{2}$ with duty cycles of $2 / 256,16 / 256$ and 1 respectively. The filling bits are set to be zero, so the $\mathrm{MBL}$ is not greater than $50 \%$. As shown in the figure, the BER of VOOK signal grows with increasing $\sigma_{\mathrm{x}}^{2}$ whereas the BER for ACO-OFDM first drops and then increases with increasing $\sigma_{\mathrm{x}}^{2}$. This verifies the analysis about the impact of $\sigma_{\mathrm{x}}^{2}$ on the BER. The impact of the error propagation is reflected from the performance degradation of the FRPO-OFDM signal with $D=2 / 256$. Compared with the other two signals, its BER for ACO-OFDM signal degrades dramatically when the decoding errors of the VOOK signals starts to take effect. It can be seen that for $\sigma_{\mathrm{x}}^{2}=19 \mathrm{dBm}$, its BER for the ACO-OFDM signal is approximately five times of those of the other two. Fortunately, the BER for the VOOK signal improves with increasing duty cycle. We can see that for duty cycles $D=16 / 256$ and 1 , the BERs of the ACO-OFDM signals are identical and that extremely low BERs are achieved by the VOOK signal (zeros errors detected in the simulation). In fact, a simulation not presented shows that the BERs for the ACO-OFDM signal are identical for all the possible duty cycles not less than 16/256 and that the BERs for the VOOK signals using these duty cycles are extremely low. This means that for a wide range of brightness levels, the extra information transmitted by the BCS can improve the spectral efficiency without causing noticeable performance degradation. The extremely low BER for the VOOK signal implies that higher order modulations such as $M$ PPM can be implemented in the BCS to further improve the spectral efficiency.

\section{CONCLUSION}

We have studied FRPO-OFDM for indoor dimmable VLC. The signal format is derived as a combination of an ACOOFDM signal with a VOOK sequence. We show that the resulting format can fully unitize LED's dynamic range, minimizing the nonlinear distortion in adjusting the brightness level. The detector for FRPO-OFDM is developed, which can recover information from both the BCS and the ACO-OFDM signal. The simulation results demonstrate that the desirable linear relationship exists between the MBL and the duty cycle. The simulated BER shows that the use of the informationcarrying BCS improves the spectral efficiency. It can be envisaged that, because of the extremely low BER achieved by the VOOK signals, modulations with higher order, such as $M$ PPM), can be adopted in the BCS to further improve the spectral efficiency.

\section{REFERENCES}

[1] F. Zafar, D. Karunatilaka, and R. Parthiban, "Dimming schemes for visible light communication: the state of research," IEEE Wireless Commun., vol. 22, pp. 29-35, Apr. 2015.

[2] K. Lee and H. Park, "Modulations for Visible Light Communications With Dimming Control," IEEE Photon. Technol. Lett., vol. 23, pp. 11361138, Aug. 2011.

[3] J. Armstrong and A. J. Lowery, "Power efficient optical OFDM," Electron. Lett., vol. 42, pp. 370-2, 2006.

[4] G. Ntogari, T. Kamalakis, J. W. Walewski, and T. Sphicopoulos, "Combining Illumination Dimming Based on Pulse-Width Modulation With Visible-Light Communications Based on Discrete Multitone," IEEE/OSA J. Opt. Commun. Netw., vol. 3, pp. 56-65, Jan. 2011.

[5] H. Elgala and T. D. C. Little, "Reverse polarity optical-OFDM (RPOOFDM): dimming compatible OFDM for gigabit VLC links," Optics Express, vol. 21, pp. 24288-24299, Oct. 2013.

[6] M. Tahar, T. Q. Wang, M. F. G. Medina, O. Gonzalez, and J. Armstrong, "Experimental Demonstration of Diversity Combining for Asymmetrically Clipped Optical OFDM," IEEE Commun. Lett., vol. 20, pp. 906-909, May 2016.

[7] L. Wu, Z. Zhang, J. Dang, and H. Liu, "Adaptive Modulation Schemes for Visible Light Communications," IEEE/OSA J. Lightw. Technol., vol. 33, pp. 117-125, Jan. 2015.

[8] I. S. Gradshteyn and I. M. Ryzhik, Table of Integrals, Series, and Products, 7th ed. San Diego, CA: Academic, 2007. 